\title{
Physical and in silico approaches identify DNA-PK in a Tax DNA-damage response interactome
}

\author{
Emad Ramadan1, Michael Ward2,3, Xin Guo ${ }^{3}$, Sarah S Durkin ${ }^{3,7}$, \\ Adam Sawyer ${ }^{3}$, Marcelo Vilela ${ }^{4}$, Christopher Osgood ${ }^{5}$, Alex Pothen ${ }^{6}$ and \\ Oliver J Semmes*2,3
}

\begin{abstract}
Address: ${ }^{1}$ Department of Computer Science, Old Dominion University, Norfolk, VA, USA, ${ }^{2}$ George L. Wright Center for Biomedical Proteomics, Eastern Virginia Medical School, Norfolk, VA, USA, ${ }^{3}$ Department of Microbiology and Molecular Cell Biology, Eastern Virginia Medical School, Norfolk, VA, USA, ${ }^{4}$ Laboratorio do Cancer, Univeridade Federal de Vicosa, Minas Gerais, Brazil, ${ }^{5}$ Department of Biology, Old Dominion University, Norfolk, VA, USA, ${ }^{6}$ Department of Computer Sciences and Computing Research Institute, Purdue University, West Lafayette IN, USA and ${ }^{7}$ Department of Exploratory Biology, Pfizer Global Research and Development, La Jolla, CA, USA

Email: Emad Ramadan - eramadan@cs.odu.edu; Michael Ward - wardmd@evms.edu; Xin Guo - guox@evms.edu; Sarah S Durkin - sjsdurkin@yahoo.com; Adam Sawyer - swayerca@evms.edu; Marcelo Vilela - marcelo@ufv.br;

Christopher Osgood - cosgood@odu.edu; Alex Pothen - apothen@purdue.edu; Oliver J Semmes* - semmesoj@evms.edu

* Corresponding author
\end{abstract}

Published: 15 October 2008

Retrovirology 2008, 5:92 doi:10.1186/1742-4690-5-92

This article is available from: http://www.retrovirology.com/content/5/I/92

(c) 2008 Ramadan et al; licensee BioMed Central Ltd.

This is an Open Access article distributed under the terms of the Creative Commons Attribution License (http://creativecommons.org/licenses/by/2.0), which permits unrestricted use, distribution, and reproduction in any medium, provided the original work is properly cited.
Received: 26 June 2008

Accepted: 15 October 2008

\begin{abstract}
Background: We have initiated an effort to exhaustively map interactions between HTLV-I Tax and host cellular proteins. The resulting Tax interactome will have significant utility toward defining new and understanding known activities of this important viral protein. In addition, the completion of a full Tax interactome will also help shed light upon the functional consequences of these myriad Tax activities. The physical mapping process involved the affinity isolation of Tax complexes followed by sequence identification using tandem mass spectrometry. To date we have mapped 250 cellular components within this interactome. Here we present our approach to prioritizing these interactions via an in silico culling process.
\end{abstract}

Results: We first constructed an in silico Tax interactome comprised of 46 literature-confirmed protein-protein interactions. This number was then reduced to four Tax-interactions suspected to play a role in DNA damage response (Rad5I, TOPI, Chk2, 53BPI). The first-neighbor and secondneighbor interactions of these four proteins were assembled from available human protein interaction databases. Through an analysis of betweenness and closeness centrality measures, and numbers of interactions, we ranked proteins in the first neighborhood. When this rank list was compared to the list of physical Tax-binding proteins, DNA-PK was the highest ranked protein common to both lists. An overlapping clustering of the Tax-specific second-neighborhood protein network showed DNA-PK to be one of three bridge proteins that link multiple clusters in the DNA damage response network.

Conclusion: The interaction of Tax with DNA-PK represents an important biological paradigm as suggested via consensus findings in vivo and in silico. We present this methodology as an approach to discovery and as a means of validating components of a consensus Tax interactome. 


\section{Background}

Human T-cell Leukemia Virus type 1(HTLV-1) is the causative agent of Adult T-cell Leukemia (ATL), HTLV-1 Associated Myelopathy/Tropical Spastic Paraparesis (HAM/ TSP) as well as other subneoplastic conditions [1-5]. Although the development of ATL is the culmination of complex events, it appears that the viral oncogene product, Tax, may provide the impetus for the transformation process. This protein has been studied extensively since 1982 when Tax was discovered to be a transactivator of the cognate viral promoter [6]. Since that time many activities and subsequent functions have been assigned to the Tax protein [7-9]. The critical importance of this protein to human disease makes it a fascinating protein as a research target; however, the result of such focused research efforts has been thousands of articles and a healthy dose of controversy. These qualities also make Tax an ideal candidate for the development of a complete list of interacting proteins as an effort to define potential protein functions.

There have been a number of published accounts of cellular proteins that bind to Tax. For example, Jin et al described the binding of Tax to MAD1 as a result of a comprehensive yeast two-hybrid approach [10]. Immunoprecipitation and western analysis has been used to identify specific Tax-protein interactions, for example IKK $\gamma$ [11,12], CRM1 [13], Dlg1 [14] and components of the APC $[15,16]$. Recently, Kashanchi and co-workers conducted a major effort using 2D gel separation followed by MALDI-MS to identify a 32-member Tax interactome [17]. A combined listing of Tax binding proteins with accompanying literature citations can be found by visiting the publicly accessible Tax website http://htlv-tax.com.

As data accumulates regarding Tax-protein interactions, a system for analysis and validation of these interactions is needed. This is especially true given the exponential increase in technical ability to identify protein-protein interactions, compounded by the inherent increases in false-positives (protein-protein interactions of no functional consequence). We describe a two-pronged approach for identification and selection of functionally significant Tax-protein interactions. The study begins with the construction of a comprehensive physical interactome using affinity isolation of Tax complexes coupled to MS/ MS analysis. Next, we utilized knowledge gained in existing literature that defined a physical interaction between Tax and a cellular protein, to comprise an in silico Tax interactome. This interactome was then restricted to proteins with a putative role in DNA repair response. The final steps expanded the in silico interactions into a nearest neighbor network to identify groups of proteins with greatest functional impact to DNA repair response. Our analysis identified DNA-PK as a top candidate protein for further analysis into the mechanism of action for Taxinduced defects in the cellular DNA damage repair response.

\section{Results \\ Assimilation of an interaction database for Tax}

We conducted a manual literature search for articles with reference to "Tax Interaction". This list of research articles was then limited to those that could be manually confirmed as containing evidence of Tax binding via physical interaction. The manual filtering resulted in a confirmed list of 67 proteins (see Table 1). As we have alluded to earlier, Tax has many putative functions but for this exercise we have limited our analysis to the DNA damage repair response. Thus, we asked which of these known protein interactions has a known function that would potentially impact the cellular DNA repair response process. Our analysis suggested a starting point of four confirmed Taxbinding proteins; Rad51, TOP1, Chk2, and 53BP1.

\section{Construction of a physical Tax interactome map}

Our approach to defining the physical Tax interactome began with the selective isolation of Tax-containing multiprotein complexes from mammalian cells. The isolation of multi-protein complexes was facilitated by the use of affinity tagged Tax protein. The S-Tax-GFP vector expresses full length TAX protein fused to amino-terminal $\mathrm{His}_{6}$ and S-tags, and carboxyl-terminal GFP protein. A critical property in such a system is the recapitulation of Tax-associated activity in the fusion protein. We have previously demonstrated that the expressed S-Tax fusion protein is fully functional when compared to wild type Tax protein $[18,19]$. The S-Tax-GFP vector was transiently transfected into $293 \mathrm{~T}$ cells, and the expression of GFP used to assess correct cellular localization and to monitor the transfection efficiency. The S-Tax-GFP protein was purified on Sagarose beads and incubated with Jurkat nuclear extracts. We used the nuclear extracts to increase the relative abundance of Tax binding proteins to Tax. A series of preliminary experiments were conducted in order to titer the best proportions between nuclear lysate concentration and the amount of Tax such that the Tax protein concentration does not either overwhelm the binding partners or disappear from the complex. In an effort to increase the binding specificity of Tax associated proteins, we pre-incubated the nuclear lysate with the S-agarose beads as a "pre-clear" step. This resulted in a significant reduction of nonspecific protein hits such as HSP's and common nuclear structural proteins like tubulin and actin. The resulting isolated protein complexes were then trypsinized and subjected to LCMS/MS analysis. When each of the three experimental runs was analyzed individually and then compared, we observed that $86 \%$ of the proteins were present on all three runs. The control experiments with the S-GFP protein alone resulted in a list of approximately 25 proteins 
Table I: Tax interacting proteins

\begin{tabular}{|c|c|c|c|}
\hline Tax interacting protein & Evidence for interaction & Alternate names & Reference \\
\hline PCAF & GST pulldown; co-IP & p300/CBP-associated factor & Jiang H, MCB I999 I9(12):8।36-45 \\
\hline PSAP & GST pulldown & Sap-I & Shuh M, J. Virol 2000 74(23): I I 394 \\
\hline ELKI & GST pulldown & ETS family & Shuh M, J. Virol 2000 74(23): I I394 \\
\hline SRF & GST pulldown & serum response factor & Shuh M, J. Virol 2000 74(23): I I 394 \\
\hline SUV39HI & GST pulldown; co-IP & KMTIA & Kamoi K, Retrovirology 2006 3:5 \\
\hline ATF4 & yeast two hybrid; GST pulldown & TAXREB67, CREB-2 & Reddy TR, Oncogene I997 I4(23):2785 \\
\hline MSX2 & co-IP & CRS2, FPP, HOX8, MSH, PFM & Twizere JC, JBC 2005 280(33):29804 \\
\hline ZFP36 & GST pulldown; co-IP; Colocalization & tristetraprolin, TTP, NUP475 & Twizere JC, JNCI 2003 95(24): 1846 \\
\hline CREBBP & GST pulldown; co-IP; Colocalization & CREB binding protein, CBP & Bex F, MCB I 998 I8(4):2392 \\
\hline p300 & GST pulldown; co-IP; colocalization & p300, KAT3B & Bex F, MCB I 998 I8(4):2392 \\
\hline MAP3KI & co-IP & MEKK, MAPKKKI & Yin MJ, Cell 1998 93(5):875 \\
\hline ACTL6A & CO-IP & BAF53, Arp4, INO80K & Wu K, JBC 2004 279(I):495 \\
\hline SMARCEI & CO-IP & BAF57, SWI/SNF related & Wu K, JBC 2004 279(I):495 \\
\hline SMARCCI & Co-IP & BAFI55, SWI/SNF related & Wu K, JBC 2004 279(I):495 \\
\hline BRGI & Co-IP & SMARCA4, SWI/SNF related & Wu K, JBC 2004 279(I):495 \\
\hline RAD5I & CO-IP & BRCC5 & Wu K, JBC 2004 279(I):495 \\
\hline RAG2 & CO-IP & & Wu K, JBC 2004 279(I):495 \\
\hline Actin & Co-IP & ACTA & Wu K, JBC 2004 279(I):495 \\
\hline CDK2 & co-IP & & Wu K, JBC 2004 279(I):495 \\
\hline CDC42 & Co-IP & G25K & Wu K, JBC 2004 279(I):495 \\
\hline RHOA & co-IP & & Wu K, JBC 2004 279(I):495 \\
\hline $\mathrm{RACl}$ & co-IP & TC-25, p2I-Racl & Wu K, JBC 2004 279(I):495 \\
\hline GSN & CO-IP & gelsolin & Wu K, JBC 2004 279(I):495 \\
\hline RASA2 & Co-IP & GAPIM & Wu K, JBC 2004 279(I):495 \\
\hline TAXIBPI & $\begin{array}{l}\text { yeast two hybrid, GST pulldown, Co- } \\
\text { localisation }\end{array}$ & TXBPI5I, CALCOCO3 & Reddy TR, PNAS 95(2): 702 \\
\hline CHEK2 & Co-IP, co-localization & CDSI, CHK2 & Haoudi A, JBC 2003 278(39):37736 \\
\hline RBI & GST pulldown & retinoblastoma I & Kehn K, Oncogene 2005 24(4):525 \\
\hline CCND2 & in vitro binding & Cyclin D2 & Fraedrich K, Retrovirology 2005 2:54 \\
\hline CDK4 & in vitro binding, mammalian two hybrid & PSK-J3 & Fraedrich K, Retrovirology 2005 2:54 \\
\hline IKBKB & co-IP & IKK-beta, IKK2, FKBIKB & Harhaj EW, JBC 274(33):2291। \\
\hline IKBKG & co-IP & IKK-gamma, NEMO, FIP3 & Harhaj EW, JBC 274(33):2291I \\
\hline CREBI & Co-IP & & Zhao LJ, PNAS 89(I5):7070 \\
\hline MADI & yeast two hybrid & TXBPI8I, MADILI, PIG9 & Jin DY, Cell 93(I):8I \\
\hline $\mathrm{CDC} 27$ & co-IP & APC3 & Liu B, PNAS 2005 102(I):63 \\
\hline CDC20 & co-IP & p55CDC, CDC20A & Liu B, PNAS 2005 102(I):63 \\
\hline RELA & co-IP & NFKB3; p65 & Lacoste, Leukemia I994 8 Suppl I:S7I \\
\hline NFYB & yeast two hybrid; GST pulldown; co-IP & CBF-A, HAP3 & Pise-Masison CA, MCB 1997 17(3):1236 \\
\hline NFKBI & co-IP & KBFI, pl05 & Beraud C, MCB I994 I4(2): 1374 \\
\hline RAN & GST pulldown; co-IP; Colocalization & ARA24, TC4, Gspl & $\begin{array}{l}\text { Peloponese JM, PNAS } 2005 \\
\text { 102(52): } 18974\end{array}$ \\
\hline RANBPI & GST pulldown; co-IP; Colocalization & HTF9A & $\begin{array}{l}\text { Peloponese JM, PNAS } 2005 \\
\text { 102(52): } 18974\end{array}$ \\
\hline CEBPB & GST pulldown & LAP, CRP2, NFIL6, TCF5 & Tsukada J, Blood 1997 90(8):3142 \\
\hline TBP & GST pulldown & TFIID & Caron C, EMBO J 1993 I2(II):4269 \\
\hline TAFII & GST pulldown; co-IP & TAF(II)28, RNA polymerase II & Caron C, PNAS 1997 94(8):3662 \\
\hline HDACI & co-IP, GST pulldown & HDI, GON-IO & Ego T, Oncogene 2002 2I(47):724I \\
\hline ATF5 & yeast two hybrid, co-IP & ATFx & Forgacs E, J Virol 2005 79(I I):6932 \\
\hline NRFI & GST pulldown & EWG, ALPHA-PAL & $\begin{array}{l}\text { Moriuchi M, AIDS Res Hum Retroviruses } \\
\text { I999 I5(9):82 I }\end{array}$ \\
\hline CDK9 & GST pulldown; co-IP & PITALRE, C-2k, TAK & Zhou M, J Virol 2006 80(10):478I \\
\hline MAGI3 & co-IP; colocalization & & Ohashi M, Virology 2004 320(I):52 \\
\hline DNAJA3 & GST pulldown; & TIDI, hTid-I & Cheng H, Curr Biol 200 I II(22): I77I \\
\hline HSPA2 & GST pulldown; Colocalization & HSP70-2 & Cheng H, Curr Biol 200I II(22): I77I \\
\hline HSPAIB & GST pulldown; Colocalization & HSP70-2 & Cheng H, Curr Biol 200 I I I(22): I77। \\
\hline TOPI & yeast two hybrid; co-IP & DNA topoisomerase I & Suzuki T, Virology 2000 270(2):291 \\
\hline CHUK & co-IP & IKK-alpha, IKKI, IKKA & Chu ZL, JBC 1999 274(22): 15297 \\
\hline SPII & GST pulldown & pI6INK4A; MTSI, pI9ARF & Tsukada J, Blood I 997 90(8):3142 \\
\hline CDKN2A & GST pulldown; co-IP & pI6INK4A; MTSI, pI9ARF & Suzuki T, EMBO J 1996 I5(7):1607 \\
\hline GTF2AI & yeast two-hybrid; GST-pulldown; co-IP & TFIIA & Clemens KE, MCB I996 I6(9):465 \\
\hline CDKNIA & CO-IP & P2ICIPI/WAFI, CAP20 & Haller K, MCB 2002 22(I0):3327 \\
\hline
\end{tabular}


Table I: Tax interacting proteins (Continued)

\begin{tabular}{|c|c|c|c|}
\hline NFKB2 & CO-IP & LYT-10 & Murakami T, Virology 1995 206(2):1066 \\
\hline VACI4 & CO-IP & TAXIBP2; TRX & Mireskandari A, BBA I996 I306(I):9 \\
\hline GPS2 & yeast two hybrid; GST pulldown & TXBP3I & Jin DY, JBC I997 272(4I):258I6 \\
\hline CCND3 & CO-IP & Cyclin D3 & Haller K, MCB 2002 22(I0):3327 \\
\hline PSMB4 & yeast two hybrid; co-IP & HN3 & Haller K, MCB 2002 22(I0):3327 \\
\hline PSMA4 & yeast two hybrid; co-IP & HC9; PSC9 & Rousset R, Nature I996 38I(6580):328 \\
\hline CARMI & GST pulldown; co-IP; Colocalization & PRMT4 & Jeong SJ, J Virol 2006 80(20): 10036 \\
\hline GNB2 & yeast two hybrid; co-IP; Colocalization & transducin beta chain 2 & Twizere JC, Blood 2007 109(3): 105 I \\
\hline GNB5 & co-IP; colocalization & GB5 & Twizere JC, Blood 2007 109(3):105। \\
\hline GNBI & co-IP; colocalization & transducin beta chain I & Twizere JC, Blood 2007 I09(3): 105। \\
\hline ILI6 & co-IP, colocalization & LCF & Wilson KC, Virology 2003 306(I):60 \\
\hline PPP2CA & co-IP, GST pulldown & PP2A catalytic subunit & Fu DX, JBC 2003 278(3):1487 \\
\hline MAP3KI4 & co-IP & NIK & Xiao G, EMBO J 2001 20(I0):6805 \\
\hline TP53BPI & co-IP, colocalization & 53BPI, p202 & Haoudi A, JBC 2003 278(39):37736 \\
\hline
\end{tabular}

consisting mainly of HSP's, actin and tubulin. Only $10 \%$ of these proteins were shared with the S-Tax-GFP experiments.

One approach to assigning value to any single proteinprotein interaction is by determining the strength of interaction. A comparable evaluation in mass spectrometry would be measurements that imply the relative sequence coverage of a particular protein within a complex. The number of peptides with sequence unique to the protein (unique peptides), the sum of the relevant peptide confidence scores (protein score), the percentage of sequence coverage (coverage) and the relative abundance of predicted peptides from a protein (emPAI) were used for ranking the Tax-binding protein identities. Such confidence values would be directly influenced by the amount of measurable protein and indirectly influenced by strength of binding. Thus, we combined the data, in which the Tax interactome was analyzed as described above, from three separate experimental runs into one data set. Each of the LC-MS/MS runs contained approximately 23,000 scans. The top 5 protein "hits" as determined via multiple measures of confidence are shown in table 2. This analysis resulted in the identification of a novel interaction between Tax and DNA-PK. We note that one possible explanation for our approach uniquely identifying DNA-PK is the enrichment of nuclear proteins in the binding reaction.

\section{Defining first neighbor interactions of the known Tax- binding proteins}

In this section we conducted a query for immediate binding partners of a selected group of known Tax-binding proteins. Our starting group of Tax-binding proteins, Rad51, TOP1, CHEK2 (Chk2), and TP53BP1 (53BP1), known to play a role in the DNA repair response, was referred to as the set $\mathrm{C} 1$. The goal was to carefully extend the four protein dataset outward to include the first neighbors of known Tax-binding proteins. We then created a network consisting of the first neighbor interactions of these four proteins with the world of proteins within the HRPD, which we call G1 = 1NN (C1). This sub-network, G1, consists of a set of 50 proteins involved in 112 interactions as shown in figure 1 . The G1 sub-network has a diameter of 5, and average path length of 2.7 , which are consistent with a small-world network.

Several features in the network G1 and other sub-networks of G1 described below, suggest a significant role for PRKDC(DNA-PKcs). The maximum core (a group of proteins with the most intra-group interactions) of G1 is 6 , and DNA-PKcs is a member of the 5-core; the 5-core is a highly interacting group of 12 proteins (DNA-PKcs, TOP1, PCNA, RPA1, DDX9, CDK4, CDKN1A (p21), CDK5, ADPRT (PARP), XRCC5 (Ku70), XRCC6 (Ku86), NCOA6 (TRBP)), all of which are related to the DNA-repair process. Interestingly 6 of these 12 proteins (DNA-PKca, TOP1, DDX9, ADPRT, XRCC5, XRCC6) were also among the Tax-binding proteins observed in the mass spectrome-

Table 2: Tax binding proteins sorted by number of unique peptides

\begin{tabular}{lllll}
\hline Protein & Unique peptides & Protein score & Coverage & emPAl \\
\hline DNA-dependent Protein Kinase & 25 & 1391 & $9 \%$ & 0.27 \\
Vimentin & 11 & 1387 & $44 \%$ & 7.54 \\
Gamma interferon-inducible protein & 19 & 1116 & $24 \%$ & 1.7 \\
PARP & 15 & 1414 & $34 \%$ & 1.78 \\
H2A.I & 7 & 569 & $30 \%$ & 1.25 \\
\hline
\end{tabular}




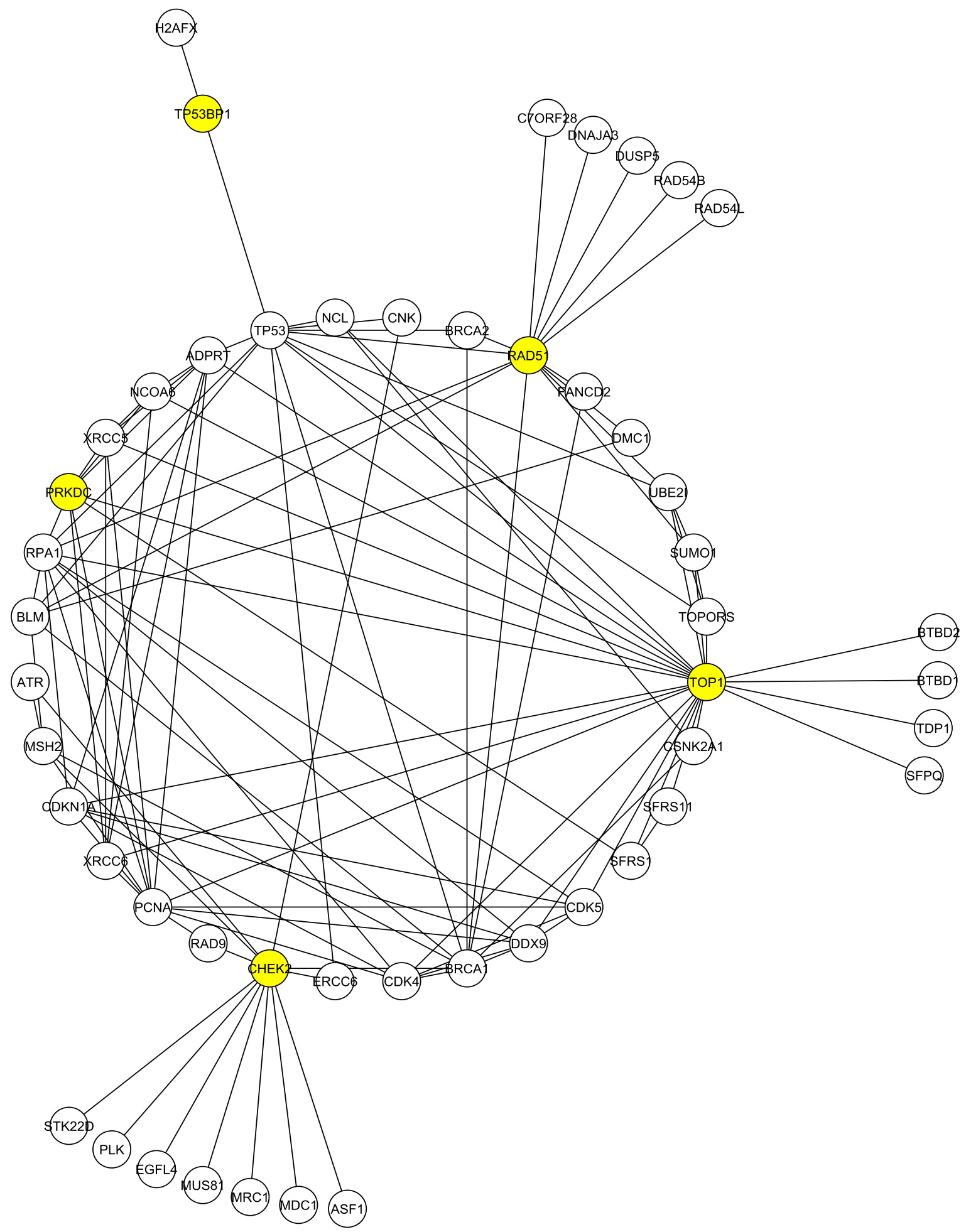

Figure I

The GI first neighborhood network for Rad5 I, TOPI, Chk2 and 53BPI. The four initial proteins (yellow) were used to generate a network via interrogation of the Human Protein Reference Database. Protein-protein interactions are indicated by lines. Proteins with two or more shared interactions will form a core. PRKDC (DNA-PK) is also highlighted. 
try analysis. We also note that active DNA-PK consists of the catalytic subunit (DNA-PKcs) and the two regulatory subunits (Ku70 and Ku86) each of which is a member of this highly interactive core. Furthermore, DNA-PKcs ranks eighth in degree (the number of interactions) and in the top $30 \%$ in two centrality measures (betweenness and closeness).

We next considered the structure of the G1 sub-network after the removal of the four initial Tax-binding proteins comprising C1. This would allow for an assessment of the degree and centrality of neighbors without interference from the original four proteins. The largest connected component of the resulting network consisted of 29 proteins and 60 interactions as shown in figure 2. This network has a diameter of 6 and a small average path length of 2.6. In this sub-network, DNA-PKcs is among the top six proteins in degree and betweenness centrality. Thus, the critical role of DNA-PKcs as determined through our clustering process is independent of the presence of the four initial proteins.
We then created a sub-network of G1 restricted to those involved in DNA repair response, referred to as G1*. Specifically, we removed those proteins that lacked the primary function of DNA repair as listed in the HRPD. This network consisted of 26 proteins and 42 interactions as shown in figure 3 . The $\mathrm{G} 1$ * network has a diameter of 5 and an average path length of 2.5. In this restricted network, DNA-PKcs ranks fourth in degree and ninth in betweenness centrality. The maximum core of this network is the 4-core, which consists of six proteins of which DNA-PKcs is a member (DNA-PKcs, PCNA, PARP, Ku70, Ku86, and TRBP). Thus, DNA-PKcs demonstrates an increased rank when consideration is refocused toward protein interactions involved in DNA damage response.

\section{Definition of the second neighbors of $\mathrm{CI}$ refined to DNA repair}

In our next exercise, we attempt to assign value to the proteins identified in the prior networks by examining their context in the "larger world" of second neighbors. Our assumption was that key proteins from the first neighbor analysis should retain their central role as defined by

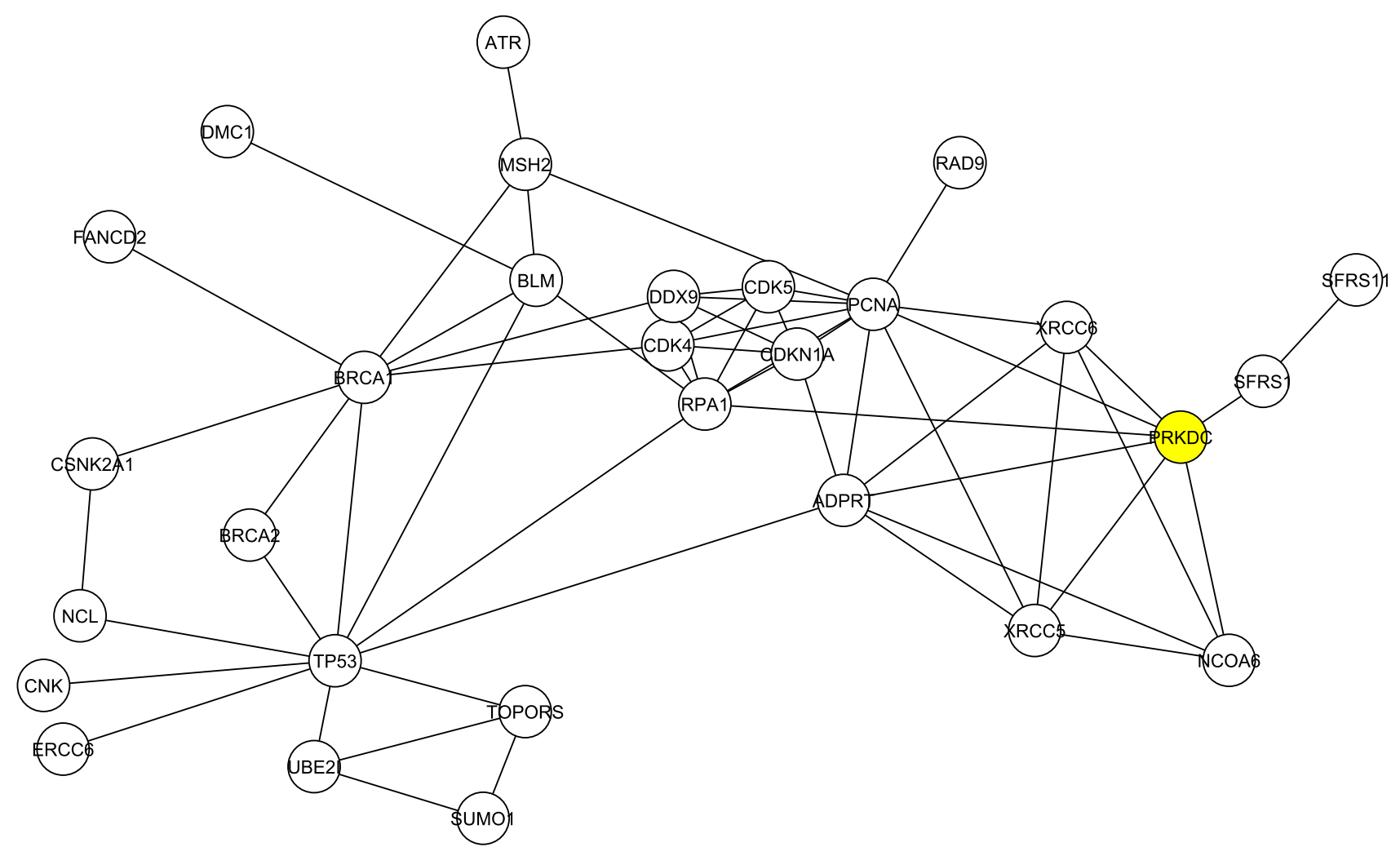

Figure 2

The largest interacting network remaining in GI after removal of Rad5 I, TOP I, Chk2 and 53BP I. The components that populated the first neighborhood network were depleted of rad5 I, topl, chk2 and 53bpl. The remaining components with the highest degree of interaction are shown. DNA-PK (PRKDC) is indicated (yellow). 


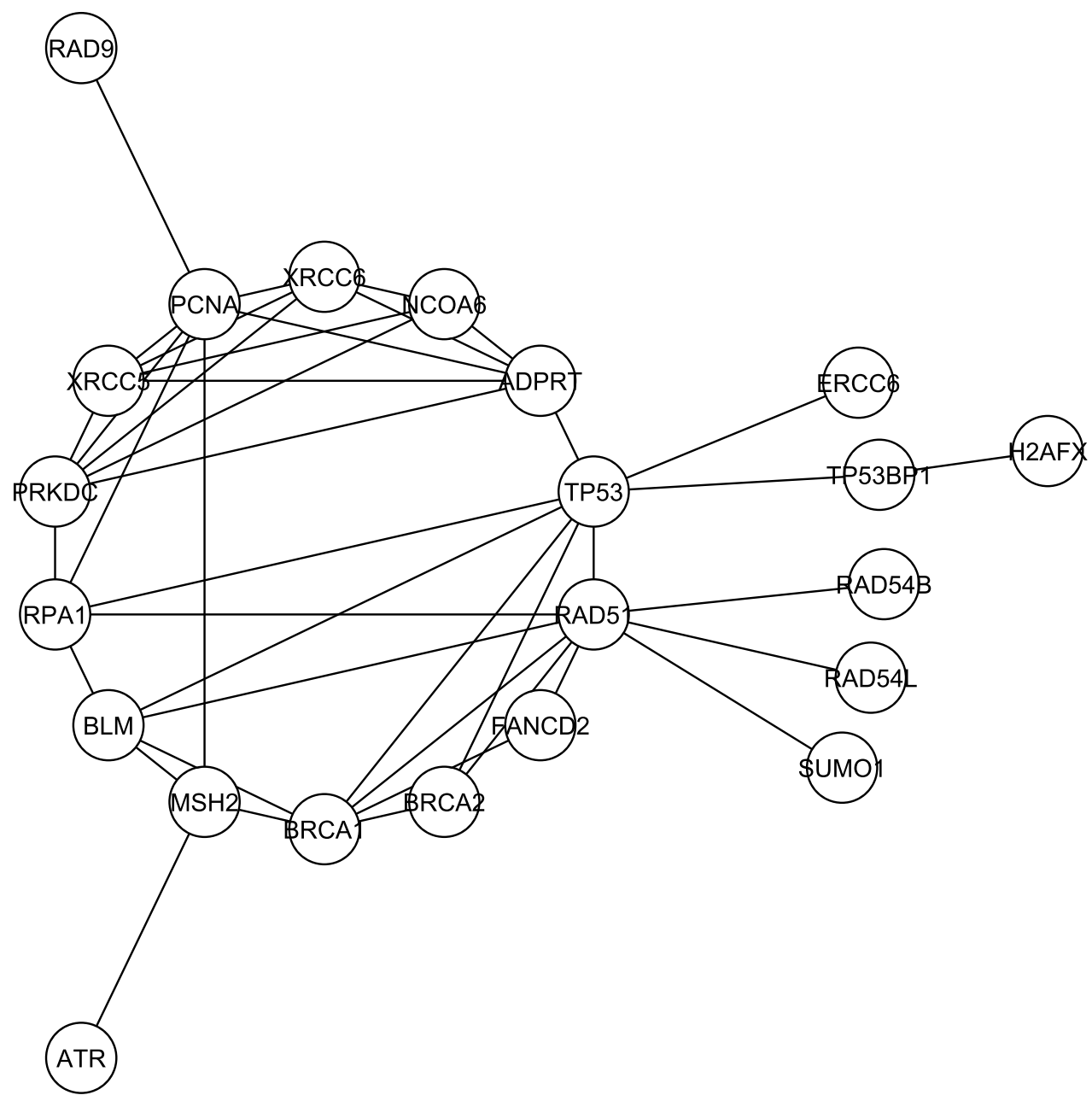

\section{Figure 3}

The GI* first neighborhood network restricted to proteins documented to play a role in the DNA-repair response. The components of the entire first neighborhood network were filtered to remove those not known to have a role in the DNA-repair response. The remaining components are displayed to reveal interactions and a central core.

interactions in the large second neighbor population. Specifically, in this exercise we first extend the database of Tax-interacting proteins outward to include second neighbor proteins (a protein that binds a protein that is known to bind Tax). We considered the first and second neighborhood of the initial set of proteins in $\mathrm{C} 1$, which we refer to as $\mathrm{G} 2=2 \mathrm{NN}(\mathrm{C} 1)$. The G2 network consisted of 667 proteins and 3827 interactions. From the proteins in the G2 network, we created a smaller network by restricting to proteins involved in DNA repair, and refer to this sub-network as G2* There were 114 proteins in G2*. Once this group is developed we use a clustering analysis in an attempt to identify the presumed most critical members of the Tax-interacting world restricted to DNA repair response proteins. The clustering process ranks components of the network based upon the intra-group interac- tions. We show the 3-core of the G2* network, which consists of 54 proteins, in figure 4 . All 3-core proteins will have three or more interactions in order to be included in the network. By application of our clustering approach, we expose the structure of this subnetwork. It consists of five clusters of proteins, with the largest cluster having 22 proteins, and the smallest cluster consisting of 3 proteins. Adding proteins of lower degree clearly generates a larger G2* network, but did not change the integrity of the structure of the network (data not shown). We can also observe from the clustering that three proteins, DNA-PKcs, PCNA, and P53 (TP53) link the various clusters to each other. We call these three proteins "bridges", since they connect the different clusters together. Hence, DNA-PKcs is a bridge protein in this second neighborhood network that links 


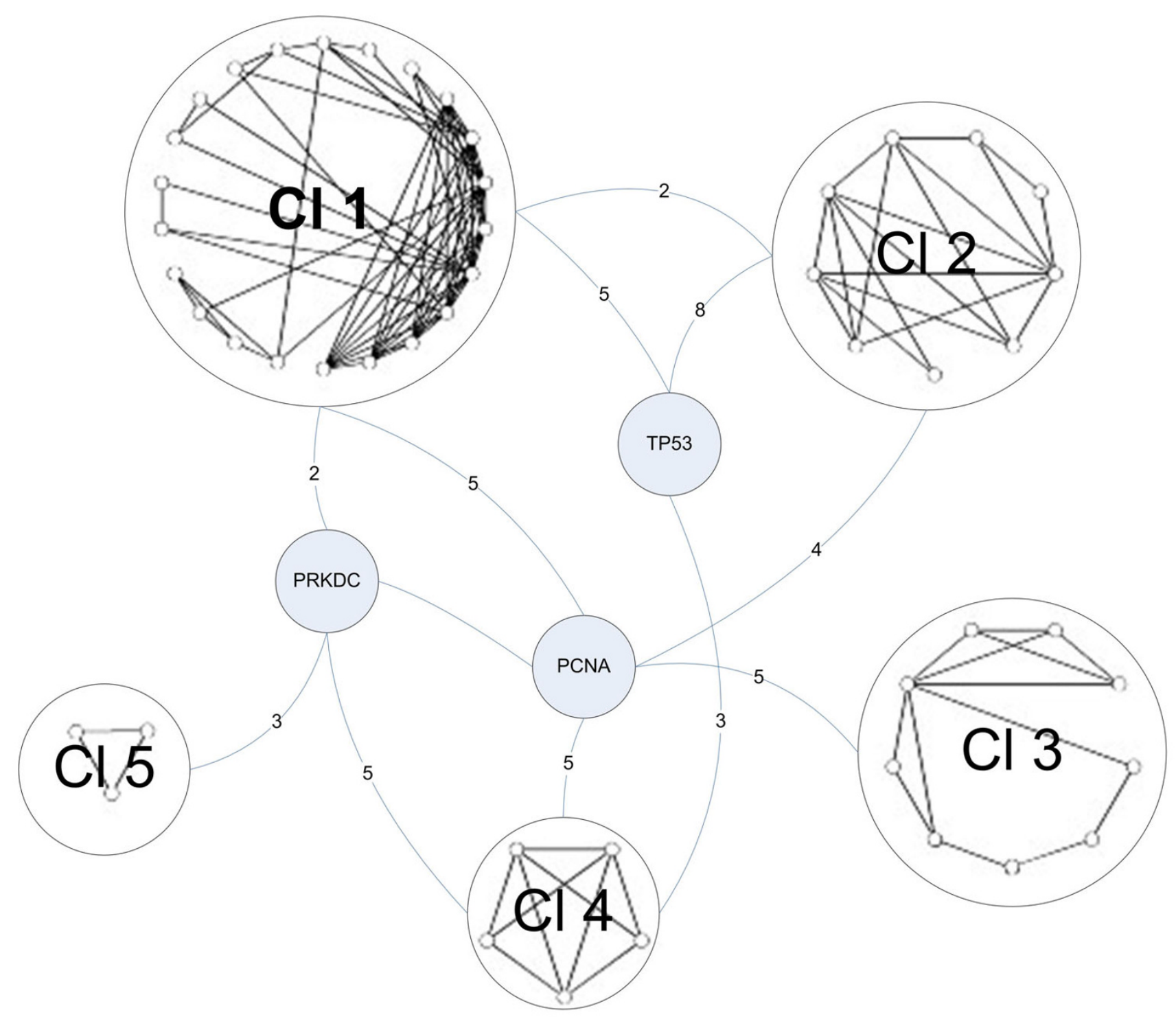

Figure 4

The 3-core representation of the G2* second neighborhood network restricted to DNA damage repair response. Shown is the result of clustering the components of the G2* second neighborhood network arising from the original four Tax binding proteins known to be involved in the cellular DNA damage response. There are five clusters with three bridge proteins; DNA-PK is one of the bridge proteins. For clarity in drawing the network, we do not show edges from these three proteins to the individual proteins in the clusters. The numbers on the edges from these proteins to the clusters count the number of edges from each protein to proteins in each cluster.

clusters 1, 4, and 5, and is also linked to the bridge protein PCNA.

The five clusters depicted in figure 4 , anchored to the three prominent bridge proteins (TP53, PCNA and PRKDC), include proteins that play key roles in DNA repair, stressinduced signaling pathways and cell cycle controls. In general, these proteins are discretely associated with the clusters. For example, Cluster 1 includes four members of the Fanconi anemia complementation group (FANCA, D2, E and G). FANC genes mediate a stress related signaling pathway that allows a normal cell to surmount certain types of damage induced in DNA, principally interstrand crosslinks [20]. In contrast, Cluster 2 includes key genes whose proteins mediate cell cycle arrest in response to genotoxic and other cellular stresses. Thus, if these protein 
interactions reflect a true subset of the proteins that are directly, or indirectly, affected by Tax-1, then this key viral protein has command over some of the principal cellular stress response pathways that might otherwise inhibit cell growth following HTLV1 infection.

\section{Endogenous DNA-PK co-precipitates with affinity isolated Tax}

As a final verification of the binding between Tax and DNA-PKcs, we performed an affinity pull-down of endogenous cellular Tax protein complexes. In this study, we expressed either S-Tax or S-GFP via transient transfection of $293 \mathrm{~T}$ cells and normalized for S-fusion protein amount. The extracts were then isolated by affinity purification of the $S$ peptide and the complexes separated on SDS-PAGE and subjected to immunoblotting with antiDNA-PKcs. Endogenous DNA-PKcs specifically associates with the Tax containing protein complex and is detected by staining with anti-DNA-PKcs (Figure 5). These results confirm the identification of DNA-PKcs as a Tax-binding protein.

\section{Discussion}

The HTLV-1 Tax protein has been defined by the proteins with which it interacts [21]. Therefore, it stands to reason that defining the functional properties of this protein will require an understanding of which cellular proteins it interacts with. Clearly, uncovering all potential interactions will include those with functional significance. However, determining which interactions support function and which interactions are of no consequence is an obvious and critical question. We have taken the approach that if we assume that Tax impacts the DNA damage repair process, as many studies support, then those interactions that are critical to the DNA damage repair process will hold greater promise of functional significance. Given this hypothesis, we devised a computational biology approach to help define which physical interactions warrant further study.

One of the challenges in computational systems biology is to create a tool to identify functional modules and the interactions among them from large-scale protein interaction networks. There are three major clustering approaches that have been employed to identify functional modules in proteomic networks. The first approach searches for sub-graphs with specified connectivity, called network motifs, and characterizes these as functional modules or parts of them. This approach is not scalable for finding larger clusters in large-scale networks. The second approach, an example of which is work by Bader and Hogue [22], identifies a seed vertex, around which to grow a cluster. The seed vertex is identified by choosing a vertex of largest weight, where the weight of a vertex is a measure of the number of edges that join the neighbors of the ver-

\section{IP: S-beads}

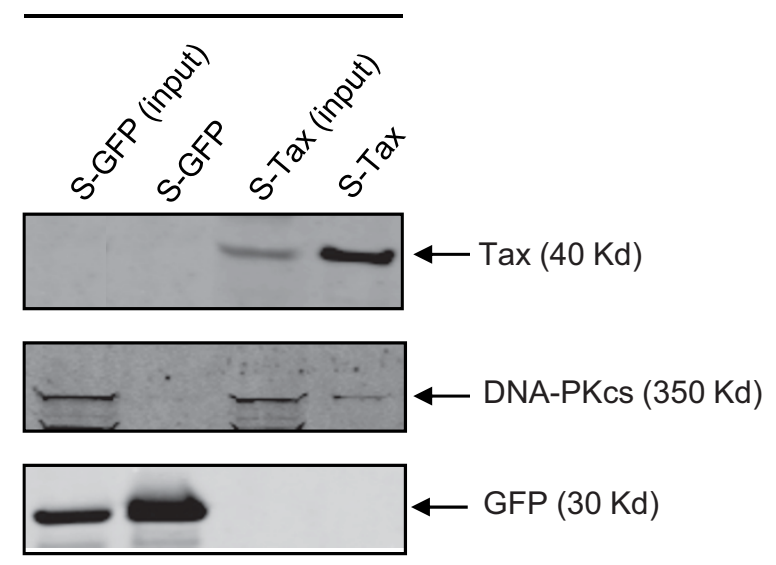

Figure 5

HTLV-I Tax binds to DNA-PKcs. The fusion proteins STax and S-GFP were isolated from 293T cells as described and analyzed for co-precipitation with DNA-PKcs. Shown is the pre-isolated total cell extract (input) for S-GFP (lane I) and S-Tax (lane 3). Also shown is the affinity purified protein complexes for S-GFP (lane 2) and S-Tax (lane 4). Experimental normalization was achieved by using equal amounts of purified protein.

tex, the clustering coefficient. A vertex in the neighborhood of a cluster is added to it as long as its weight is close (within a threshold) to the weight of the seed vertex. Once a cluster has been identified, the procedure is repeated with a vertex of largest weight that currently does not belong to a cluster as the seed vertex. However, our experience comparing this approach with the spectral algorithms we employed in this study indicates that this method is less stable (i.e., the clusters obtained depend strongly on the seed vertices chosen). We used an improved clustering method [23] to reveal proteins that form functional modules, i.e., multiple proteins involved in the same biological function. This approach was used to apply an objective measure to the functional significance of a protein. Specifically we use this to both cluster proteins into specific functional domains as well as to objectively measure each individual protein's value to that functional domain.

When we compared these results to the Tax-binding proteins generated from our physical mapping efforts, DNAPK was in the top five best represented binding proteins and occupied a top tier ranking via our functional clustering for DNA damage proteins. Clearly, DNA-PK is a critical component in cellular processes that mediate response to damage and thus the fact that our clustering analysis places high value on this protein is as much a validation 
of the process as it is novel information. However, we began with a network of known Tax-binding proteins and their neighbors and second-neighbors, and DNA-PK was selected, through our functional clustering approach, whereas other equally critical damage response proteins were not. For instance, among the PI3K protein family members ATM and ATR hold positions of prominence in the DNA damage-response arena equal to DNA-PK [24]. In fact, the three proteins are considered redundant in specific pathways and are sometimes able to substitute functionally [25-27]. However, neither of the other two proteins was reflected in the upper tier interactions when using the Tax-designated protein networks. Furthermore, ATM and ATR were not found among the list of Tax-binding proteins identified in the physical isolation of Tax complexes, again verifying the novelty of the DNA-PK finding.

This is not the first time that DNA-PK has been targeted as a cellular protein through which Tax might mediate genomic instability [28]. It is clear that DNA-PK is known to mediate functions associated with reported Tax activities. Specifically, Tax has been shown to cause constitutive activation of Chk2, a downstream target of DNA-PK [19]. DNA-PK can phosphorylate the tumor suppressor p53 at S15 and S37 [29] whereas Tax expression results in phosphorylation at S15 and S392 [30,31]. In addition, we have recently shown that Tax interaction with DNA-PK results in saturation of the damage response (manuscript submitted). Thus, the Tax-DNA-PK interaction satisfies several previous observations regarding Tax function and provides a unifying model for all of these activities. Thus, although Van et al. [32] demonstrated that the Tax-p53 nexus was intact in a DNA-PK knock-out line, it may well be worth examining this protein as a mediator of other Tax activities.

Clearly HTLV-1 Tax presents a biological model for an interesting protein with an overwhelming amount of associated published literature. A recent review by Boxus et al highlights this complexity and presents an exhaustive compilation of all known Tax-interacting proteins [33]. The growth in the Tax knowledge base requires constant surveillance and verification if this body of work is to be useful in understanding how Tax functions. Additionally, as proteomic techniques continue to mature, the data generated in experimental studies is increasing exponentially. We have described a parallel process for combining in silico analysis with experimental proteomic analysis so that information gained in each process facilitates data mining of the orthogonal process. Further building of the Tax interactome should reveal other critical proteins that play key roles in mediating the biologically significant Tax functions within the host cell.

\section{Methods \\ Cell culture and transfection}

$293 \mathrm{~T}$ cells were maintained at $37^{\circ} \mathrm{C}$ in a humidified atmosphere of $5 \% \mathrm{CO}_{2}$ in air, in Iscove's modified Dulbecco's medium supplemented with $10 \%$ fetal bovine serum and $1 \%$ penicillin-streptomycin. Transient transfections were performed by standard calcium phosphate precipitation. The plasmid used for expression of S-TaxGFP has been described previously [18]. For expression of S-Tax and S-GFP the tax or EGFP open reading frame was inserted into the SmaI site of pTriEx4-Neo (Novagen, Madison, WI). Cells were plated in 150-mm plates at $4 \times 10^{6}$ cells per plate. The following day, $20 \mu \mathrm{g}$ of plasmid DNA in $2 \mathrm{M} \mathrm{CaCl}_{2}$ and $2 \mathrm{X} \mathrm{HBS}$ were added drop wise to cells in fresh medium. Cells were incubated at $37^{\circ} \mathrm{C}$ for $5 \mathrm{~h}$ and fresh medium was added. The cells were harvested $48 \mathrm{~h}$ later.

\section{Purification of S-fusion proteins}

S-Tax-GFP, S-Tax, or S-GFP protein was isolated following a single wash with $1 \mathrm{X}$ PBS, in $500 \mu \mathrm{l}$ M-Per mammalian protein extraction reagent (Pierce, Rockford, IL) supplemented with protease inhibitor cocktail (Roche, Palo Alto, CA) and immediately frozen at $-80^{\circ} \mathrm{C}$. The cell lysate $(2.5 \mathrm{~mL})$ was incubated with $200 \mu \mathrm{l}$ bed volume of S-protein $^{\mathrm{TM}}$ agarose (Novagen, Madison, WI) for $30 \mathrm{~min}$ at room temperature as per manufacturer's suggestion. The bound S-tagged protein was then washed 3 times with 1 $\mathrm{mL}$ Bind/Wash Buffer (20 mM Tris- $\mathrm{HCl} \mathrm{pH}$ 7.5, $150 \mathrm{mM}$ $\mathrm{NaCl}, 0.1 \%$ TritonX-100).

\section{Isolation of Tax-complexes}

Freshly prepared S-Tax-GFP or S-GFP beads were washed $3 \times$ in incubation buffer (25 mM HEPES, pH 7.5, $150 \mathrm{mM}$ $\mathrm{NaCl}, 1 \% \mathrm{NP}-40,10 \mathrm{mM} \mathrm{MgCl} 2,1 \mathrm{mM}$ EDTA, 1\% glycerol) and placed on ice. A working stock of Jurkat nuclear lysate (Active Motif, Carlsbad CA) was prepared by diluting $25 \mu \mathrm{g}$ lysate to a total volume of $75 \mu \mathrm{L}$ in incubation buffer. The lysate was pre-cleared by adding $30 \mu \mathrm{L}$ of Sbead slurry and incubating on ice for 30 minutes with occasional mixing. The pre-cleared slurry was spun down at $2000 \mathrm{~g}$ for 3 minutes and the lysate $(70 \mu \mathrm{L})$ transferred to a fresh $0.5 \mathrm{ml}$ tube containing $10 \mu \mathrm{L}$ of the S-Tax-GFP or S-GFP protein bound to beads. This slurry was incubated at $4^{\circ} \mathrm{C}$ for 60 minutes on a shaker. The beads were centrifuged at $2000 \mathrm{~g}$ for 3 minutes, lysate removed, and beads washed $1 \times$ with $250 \mu \mathrm{L}$ incubation buffer followed by 4 washes with $250 \mu \mathrm{L}$ ice cold PBS.

\section{Isolation of endogenous DNA-PK-Tax protein complex}

In some cases, S-Tax or S-GFP expression plasmids were transfected into 293T and protein complexes isolated as described above from a single T75 flask. In these experiments no nuclear extracts were added. The protein lysates were subjected to purification on S-beads, $50 \mu \mathrm{L}$ of sample 
loading buffer (Bio-Rad, Hercules, CA) with $\beta$-mercaptoethanol was added to the S-bead pellet and boiled for $10 \mathrm{~min}$. The whole protein sample that was bound to the S-bead was separated by 4-12\% SDS-PAGE and analyzed by Western Blot as described below.

\section{LC-MSIMS of protein complexes}

S-Tax-GFP or S-GFP beads were washed 3X with ice cold $50 \mathrm{mM}$ ammonium bicarbonate, $\mathrm{pH} 8$ and subsequently resuspended in $50 \mu \mathrm{L}$ of $50 \mathrm{mM}$ ammonium bicarbonate, $10 \%$ acetonitrile containing $3.12 \mathrm{ng} / \mu \mathrm{L}$ sequencing grade modified trypsin (Promega Corp., Madison, WI). The digest was incubated for 6 hours at $37^{\circ} \mathrm{C}$ with occasional mixing, transferred to a $0.2 \mu \mathrm{m}$ centrifuge tube filter and spun at $5000 \mathrm{rpm}$ for 3 minutes. The flow through was recovered and peptides dried in a speed vac. Digests were resuspended in $20 \mu \mathrm{l}$ Buffer A (5\% Acetonitrile, 0.1\% Formic Acid, $0.005 \%$ heptafluorobutyric acid) and $10 \mu$ l were loaded onto a $12-\mathrm{cm} \times 0.075 \mathrm{~mm}$ fused silica capillary column packed with $5 \mu \mathrm{M}$ diameter C-18 beads (The Nest Group, Southborough, MA) using a N2 pressure vessel at 1100 psi. Peptides were eluted over 300 minutes, by applying a $0-80 \%$ linear gradient of Buffer B (95\% Acetonitrile, 0.1\% Formic Acid, 0.005\% HFBA) at a flow rate of $150 \mu \mathrm{l} / \mathrm{min}$ with a pre-column flow splitter resulting in a final flow rate of $\sim 200 \mathrm{nl} / \mathrm{min}$ directly into the source. A LTQ $^{\text {TM }}$ Linear Ion Trap (ThermoFinnigan, San Jose, CA) was run in an automated collection mode with an instrument method composed of a single segment and 5 datadependent scan events with a full MS scan followed by 4 MS/MS scans of the highest intensity ions. Normalized collision energy was set at $28 \%$, activation $\mathrm{Q}$ was 0.250 with minimum full scan signal intensity at $1 \times 10^{5}$ with no minimum $\mathrm{MS}^{2}$ intensity specified. Dynamic exclusion was turned on utilizing a three minute repeat count of 2 with the mass width set at $1.0 \mathrm{~m} / \mathrm{z}$. Protein searches were performed with MASCOT version 2.2.0 v (Matrix Sciences, London GB) using the SwissProt version 51.3 database. Parent ion mass tolerance was set at 1.5 and MS/MS tolerance $0.5 \mathrm{Da}$.

\section{Western analysis}

Total protein concentrations were determined by Protein Assay (Bio-Rad, Hercules, CA). An equal volume of sample loading buffer (Bio-Rad, Hercules, CA) with $\beta$-mercaptoethanol was added to the lysate and boiled for $5 \mathrm{~min}$. Samples were normalized to total protein and separated through a $10 \%$ SDS-polyacrylamide gel. The proteins were transferred onto Immobilon-P (Millipore, Billerica, MA) membrane using a Trans-blot SD semi-dry transfer cell (Bio-Rad, Hercules, CA) at $400 \mathrm{~mA}$ for $50 \mathrm{~min}$. Following blocking in 5\% non-fat milk in PBS/0.1\% Tween-20, blots were incubated in primary antibody overnight, followed by $1 \mathrm{~h}$ incubation in secondary horseradish-peroxidase conjugated anti-mouse or anti-rabbit antibody (Bio-Rad,
Hercules, CA). Immunoreactivity was detected via Immunstar enhanced chemiluminescence protein detection (Bio-Rad, Hercules, CA). The following primary antibodies were used in the analysis: mouse monoclonal antibody of DNA-PKcs (Upstate), 1:1000; rabbit polyclonal antibody of Tax, 1:5000; mouse monoclonal antibody of GFP (Santa Cruz), 1: 2000.

\section{Sources of data for in silico analysis}

Interaction data were gathered from three types of information sources: manual extraction from Pubmed, laboratory derived physical interactions, and protein interaction databases. In the first database source, the information was extracted by manually searching the Pubmed literature to obtain a list of known Tax binding proteins. The criterion for acceptance in this group was physical verification of binding in the referenced publication. For the second database source, the physical interactions utilized in this study were all derived from the experimental efforts described elsewhere in this article. For the final database source, we queried a human protein interaction database; The Human Protein Reference Database (HPRD) [34]. The HPRD http://www.hprd.org contains interactions of proteins in the human proteome manually extracted from the literature by expert biologists who read, interpret and analyze the published data.

\section{Terms and definitions for in silico analysis}

For our topological studies of interaction networks, we utilized a novel overlapping clustering approach [23] that exposes the modular structure of the network. We define bridges as proteins that belong to multiple clusters due to the overlap among them. We also employed centrality measures of networks known as betweenness and closeness. To define these measures, first we need to define some network concepts. The distance of a protein $v$ from another protein $w$ is the number of edges in a shortest path between them. The diameter of a network is the maximum distance between any pair of vertices. The average path length of a network is the average distance over all pairs of vertices. The closeness centrality measure for a protein, $v$, is the reciprocal of the sum of the distances of $v$ to all other proteins in the network.

The dependence of a protein $s$ on a protein $v$ is the sum over all proteins $\mathrm{t}$ in the network of the ratio of the number of distinct shortest paths between proteins $s$ and $t$ that includes $v$ as an intermediate vertex, and the number of distinct shortest paths between $s$ and $t$. The betweenness value of a protein $v$ is the sum of the dependence values of all proteins $s$ on the protein $v$. This is equivalent to the following equation for betweenness. 


$$
B(v)=\sum_{\substack{s \in v \\ s \neq v}} \sum_{\substack{t \in v \\ t \neq s, t \neq v}} \frac{\sigma_{s t}(v)}{\sigma_{s t}}
$$

Here $\mathrm{V}$ is the set of proteins in the network. The numerator in the fraction shows the number of distinct shortest paths joining $\mathrm{s}$ and t on which $\mathrm{v}$ is an intermediate vertex; the denominator is the number of distinct shortest paths joining $s$ and $t$. Further details on centrality measures are available in [35].

As in earlier work [36], we define hubs as all proteins that are ranked in the top $20 \%$ with respect to degree in the network (the number of interactions a protein is involved in). Similarly bottlenecks are all the proteins that are ranked in the top $20 \%$ of betweenness values. To calculate betweenness values for proteins, we used an algorithm provided by Yu et al. [37].

In the clustering approach to be described next, we use the concept of a $k$-core of a graph. The $k$-core of a graph is obtained by repeatedly deleting all vertices which are joined to the vertices remaining in the graph by fewer than $k$ edges. This procedure begins by deleting all vertices whose degree is less than $k$. The deletion of such vertices could decrease the degrees of the remaining vertices. If some of these vertices have degrees less than $k$, they would be deleted as well. This process is repeated until the subgraph that remains has every vertex with degree at least $k$; this subgraph is the k-core of the graph. All the deleted vertices belong to the $(k-1)$-shell. Computing the k-core of a graph helps with denoising the interaction network by removing many false positives, and also reduces the initial size of the network to be clustered. The deleted vertices will be added to the clustering obtained in a subsequent step.

\section{Spectral clustering and modules identification}

We now summarize the technique we used for clustering the protein interaction networks [23]. The protein interaction network is represented by a graph $G=(V, E)$, with the proteins constituting a set of proteins $\mathrm{V}$, and interactions constituting the set of edges $\mathrm{E}$. We obtain clusters in the interaction network by identifying a number of subgraphs of $\mathrm{G}$ that have a relatively large number of edges joining vertices in each subgraph and fewer edges to vertices outside the subgraph. We permit these clusters to overlap (have some vertices in common), since proteins have multiple functions and could be involved in more than one biological process.

The details of the clustering algorithm will be described elsewhere, but here we provide an overview. Clusters are obtained by dividing a subgraph at each step into two subgraphs based on the ratio of the number of edges that join vertices in the subgraph to the total number of edges, a measure called the cohesion of the subgraph. Given the initial graph $G$, we recursively split it into subgraphs until the value of cohesion of a subgraph is above a threshold value, or the subgraph has number of vertices fewer than a threshold size. We have used a spectral algorithm that uses the components of an eigenvector of the Laplacian matrix of the graph to divide each subgraph into two. Once the eigenvector is computed (its components correspond to the vertices of the graph), those vertices whose component values are below some specified value are included in one subgraph and the others belong to the second subgraph. The choice of the value where the split should be made is based on computing the cohesion.

We have found that the overall clustering approach described above needed to be adapted to protein interaction networks, which are small-world and modified power-law networks. Initially we decompose the vertices of the network into three sets; hubs or high degree vertices (those in the top 20\% of the degrees); low-shell vertices (vertices not in the 3-core of the network); and the residual sub-network, which forms a 3-core of the network from which the hubs have been removed. We call the last subnetwork as the local network. We have found it advantageous to cluster the local and hub sub-networks separately using the spectral clustering method described above. The clusters from both sub-networks are then merged together if a large number of edges join clusters from the two networks. We check to see if nodes that belong to a cluster are significantly connected to other clusters, and if so, they are included in such clusters as well. The statistical significance of the connections is computed using a $p$-value based on the hypergeometric distribution. Finally, the low-shell nodes are added to clusters; each such node could be added to none, one, or more than one cluster, based on whether it has a statistically significant number of connections to the clusters that have been found. If a node belongs to three or more clusters, we call it a bridge node.

\section{Competing interests}

The authors declare that they have no competing interests.

\section{Authors' contributions}

ER performed the computational experiments on the interaction networks. MW performed all mass spectrometry analysis. XG and SD conducted the Tax-DNA-PKcs binding experiments. AS contributed to the compilation of Tax binding proteins. MV was responsible for study design and interpretation of results. $\mathrm{CO}$ was involved in aspects of study design and manuscript preparation. AP designed the network algorithms and helped with the 
writing. OJ designed the study, interpreted results and contributed to manuscript preparation.

\section{Acknowledgements}

We thank Kurt Maly and Mohammed Zubair of Old Dominion University, our collaborators on the Human Virus Interactome Resource (HVIR) project, who designed a digital library for representing protein interactions involving viral and human proteins. This study was supported, in part, by the United States Public Service Grant CA076595 from the National Cancer Institute, National Institutes of Health, awarded to OJS and a multi-disciplinary research initiative grant from the Old Dominion University Research Foundation, awarded to AP, CO, and OJS.

\section{References}

I. Gessain A, Barin F, Vernant JC, Gout O, Maurs L, Calender A, de The $\mathrm{G}$ : Antibodies to human T-lymphotropic virus type-I in patients with tropical spastic paraparesis. Lancet 1985, 2:407-410.

2. Osame M, Usuku K, Izumo S, Ijichi N, Amitani H, Igata A, Matsumoto M, Tara M: HTLV-I associated myelopathy, a new clinical entity. Lancet 1986, I:1031-1032.

3. Poiesz BJ, Ruscetti FW, Gazdar AF, Bunn PA, Minna JD, Gallo RC: Detection and isolation of type $C$ retrovirus particles from fresh and cultured lymphocytes of a patient with cutaneous T-cell lymphoma. Proc Natl Acad Sci USA 1980, 77:74I5-74I9.

4. Takatsuki K: Discovery of adult T-cell leukemia. Retrovirology 2005, 2:16.

5. Yoshida M, Seiki M, Yamaguchi K, Takatsuki K: Monoclonal integration of human $T$-cell leukemia provirus in all primary tumors of adult $\mathrm{T}$-cell leukemia suggests causative role of human T-cell leukemia virus in the disease. Proc Natl Acad Sci USA 1 984, 81:2534-2537.

6. Yoshida M, Miyoshi I, Hinuma Y: Isolation and characterization of retrovirus from cell lines of human adult $T$-cell leukemia and its implication in the disease. Proc Natl Acad Sci USA 1982, 79:203|-2035.

7. Giam CZ, Jeang KT: HTLV-I Tax and adult T-cell leukemia. Front Biosci 2007, I 2: 1496-I507.

8. Marriott SJ, Semmes OJ: Impact of HTLV-I Tax on cell cycle progression and the cellular DNA damage repair response. Oncogene 2005, 24:5986-5995.

9. Peloponese JM Jr, Kinjo T, Jeang KT: Human T-cell leukemia virus type I Tax and cellular transformation. Int J Hematol 2007, 86: $101-106$.

10. Jin DY, Spencer F, Jeang KT: Human T cell leukemia virus type I oncoprotein Tax targets the human mitotic checkpoint protein MADI. Cell 1998, 93:81-91.

II. Harhaj EW, Sun SC: IKKgamma serves as a docking subunit of the IkappaB kinase (IKK) and mediates interaction of IKK with the human T-cell leukemia virus Tax protein. J Biol Chem |999, 274:229||-229|4.

12. Jin DY, Giordano V, Kibler KV, Nakano H, Jeang KT: Role of adapter function in oncoprotein-mediated activation of NFkappaB. Human T-cell leukemia virus type I Tax interacts directly with IkappaB kinase gamma. J Biol Chem 1999, 274: I7402-I7405

13. Gatza ML, Dayaram T, Marriott S): Ubiquitination of HTLV-I Tax in response to DNA damage regulates nuclear complex formation and nuclear export. Retrovirology 2007, 4:95.

14. Ishioka K, Higuchi M, Takahashi M, Yoshida S, Oie M, Tanaka Y, Takahashi S, Xie L, Green PL, Fujii M: Inactivation of tumor suppressor DIg I augments transformation of a T-cell line induced by human T-cell leukemia virus type I Tax protein. Retrovirology 2006, 3:7I.

I5. Liu B, Hong S, Tang Z, Yu H, Giam CZ: HTLV-I Tax directly binds the Cdc20-associated anaphase-promoting complex and activates it ahead of schedule. Proc Natl Acad Sci USA 2005, 1 02:63-68.

16. Merling R, Chen C, Hong S, Zhang L, Liu M, Kuo YL, Giam CZ: HTLV-I Tax mutants that do not induce $G I$ arrest are disabled in activating the anaphase promoting complex. Retrovirology 2007, 4:35.
17. Wu K, Bottazzi ME, de la Fuente C, Deng L, Gitlin SD, Maddukuri A, Dadgar S, Li H, Vertes A, Pumfery A, Kashanchi F: Protein profile of tax-associated complexes. J Biol Chem 2004, 279:495-508.

18. Durkin SS, Ward MD, Fryrear KA, Semmes OJ: Site-specific phosphorylation differentiates active from inactive forms of the human T-cell leukemia virus type I Tax oncoprotein. J Biol Chem 2006, 28 I:31705-3I7I2.

19. Gupta SK, Guo X, Durkin SS, Fryrear KF, Ward MD, Semmes OJ: Human T-cell leukemia virus type I Tax oncoprotein prevents DNA damage-induced chromatin egress of hyperphosphorylated Chk2. J Biol Chem 2007, 282:2943 I-29440.

20. Niedernhofer LJ, Lalai AS, Hoeijmakers JH: Fanconi anemia (cross)linked to DNA repair. Cell 2005, I 23: I I9I-I 198.

21. Wycuff DR, Marriott SJ: The HTLV-I Tax oncoprotein: hypertasking at the molecular level. Front Biosci 2005, 10:620-642.

22. Bader GD, Hogue CW: An automated method for finding molecular complexes in large protein interaction networks. BMC Bioinformatics 2003, 4:2.

23. Ramadan E, Osgood C, Pothen A: The architecture of a proteomic network in the yeast. Lecture Notes in Bioinformatics 2005, 3695:265-276.

24. Abraham RT: PI 3-kinase related kinases: 'big' players in stressinduced signaling pathways. DNA Repair (Amst) 2004, 3:883-887.

25. Marone R, Cmiljanovic V, Giese B, Wymann MP: Targeting phosphoinositide 3-kinase: moving towards therapy. Biochim Biophys Acta 2008, I 784: I59-185.

26. Pommier Y, Sordet O, Rao VA, Zhang H, Kohn KW: Targeting chk2 kinase: molecular interaction maps and therapeutic rationale. Curr Pharm Des 2005, I I:2855-2872.

27. Yang J, Yu Y, Hamrick HE, Duerksen-Hughes PJ: ATM, ATR and DNA-PK: initiators of the cellular genotoxic stress responses. Carcinogenesis 2003, 24:157|-I580.

28. Majone F, Luisetto R, Zamboni D, Iwanaga $Y$, Jeang $K T$ : Ku protein as a potential human T-cell leukemia virus type I (HTLV-I) Tax target in clastogenic chromosomal instability of mammalian cells. Retrovirology 2005, 2:45.

29. Lees-Miller SP, Sakaguchi K, Ullrich SJ, Appella E, Anderson CW: Human DNA-activated protein kinase phosphorylates serines $\mathbf{I}$ and 37 in the amino-terminal transactivation domain of human p53. Mol Cell Biol 1992, I 2:504I-5049.

30. Pise-Masison CA, Mahieux R, Jiang $H$, Ashcroft $M$, Radonovich $M$, Duvall J, Guillerm C, Brady JN: Inactivation of p53 by human Tcell lymphotropic virus type I Tax requires activation of the NF-kappaB pathway and is dependent on p53 phosphorylation. Mol Cell Biol 2000, 20:3377-3386.

31. Pise-Masison CA, Radonovich M, Sakaguchi K, Appella E, Brady JN: Phosphorylation of p53: a novel pathway for p53 inactivation in human T-cell lymphotropic virus type I-transformed cells. J Virol 1998, 72:6348-6355.

32. Van PL, Yim KW, Jin DY, Dapolito G, Kurimasa A, Jeang KT: Genetic evidence of a role for ATM in functional interaction between human T-cell leukemia virus type I Tax and p53. J Virol 200I, 75:396-407.

33. Boxus M, Twizere JC, Legros S, Dewulf JF, Kettmann R, Willems L: The HTLV-I Tax interactome. Retrovirology 2008, 5:76.

34. Peri S, Navarro JD, Kristiansen TZ, Amanchy R, Surendranath V Muthusamy B, Gandhi TK, Chandrika KN, Deshpande N, Suresh S, et al.: Human protein reference database as a discovery resource for proteomics. Nucleic Acids Res 2004, 32:D497-50I.

35. Brandes U: A faster algorithm for betweeness centrality. Journal of Methematical Sociology 200I, 25:163-177.

36. Barabasi $A L$, Oltvai ZN: Network biology: understanding the cell's functional organization. Nat Rev Genet 2004, 5: I 0 I-I I 3.

37. Yu H, Kim PM, Sprecher E, Trifonov V, Gerstein M: The importance of bottlenecks in protein networks: correlation with gene essentiality and expression dynamics. PLOS Comput Biol 2007, 3:e59. 